\title{
Endoscopic mucosal resection with anchoring of the snare tip: multicenter retrospective evaluation of effectiveness and safety $\square$
}

\section{(ㄷ)(우우}

\section{Authors}

Mathieu Pioche ${ }^{1,2}$, Timothée Wallenhorst ${ }^{3}$, Hugo Lepetit ${ }^{4}$, Vincent Lépilliez ${ }^{5}$, Jérôme Rivory ${ }^{1}$, Romain Legros ${ }^{4}$, Florian Rostain ${ }^{1}$, Laurent Bianchi ${ }^{4}$, Aurélie Charissoux ${ }^{6}$, Valérie Hervieu ${ }^{7}$, Maira Moreno-Garcia ${ }^{1}$, Philip Robinson $^{8}$, Jean-Christophe Saurin ${ }^{1}$, Thierry Ponchon ${ }^{1}$, Marie Viprey ${ }^{9}$, Laurent Roche ${ }^{10}$, Fabien Subtil ${ }^{10}$, Jérémie Jacques ${ }^{4}$

Institutions

1 Gastroenterology and Endoscopy Unit, Pavillon $\mathrm{H}$, Edouard Herriot Hospital, Lyon, Hospices Civils de Lyon, France

2 Inserm U1032, Labtau, Lyon, France

3 Gastroenterology and Endoscopy Unit, Pontchaillou University Hospital, Rennes, France

4 Gastroenterology and Endoscopy Unit, Dupuytren University Hospital, Limoges, France

5 Gastroenterology and Endoscopy Unit, Mermoz Private Hospital, Lyon, France

6 Pathology Division, Dupuytren University Hospital, Limoges, France

7 Pathology Division, Edouard Herriot Hospital, Lyon, France

8 Délégation de la Recherche Clinique et de l'Innovation, Hospices Civils de Lyon, France

9 Pôle de Santé Publique, Hospices Civils de Lyon, Lyon, France; Univ Lyon, Université Claude Bernard Lyon 1, HESPER EA 7425, Lyon, France

10 Service de Biostatistique, Hospices Civils de Lyon, Lyon, France; Univ Lyon, Université Lyon 1, CNRS, Laboratoire de Biométrie et Biologie Évolutive UMR 5558, Villeurbanne, France

submitted 7.12 .2018

accepted after revision 9.7.2019

\section{Bibliography}

DOI https://doi.org/10.1055/a-0990-9068 |

Endoscopy International Open 2019; 07: E1496-E1502

(c) Georg Thieme Verlag KG Stuttgart · New York

eISSN 2196-9736

Corresponding author

Mathieu Pioche, MD PhD, Gastroenterology and Endoscopy Unit - Digestive Disease Department, H Pavillon - Edouard Herriot Hospital, 69437 Lyon Cedex, France

Fax: +33-4-72110147

mathieu.pioche@chu-lyon.fr

\section{ABSTRACT}

Background Endoscopic mucosal resection (EMR) with snare is the recommended technique to resect non-invasive colorectal neoplastic lesions between 10 and $30 \mathrm{~mm}$ in diameter. The objective of EMR is to resect completely the neoplastic tissue en bloc and preferably with free margins (R0), avoiding recurrences. Anchoring the tip of the snare in the submucosa is a technical trick that allows snare sliding to be reduced and larger pieces to be caught. The aim of the present study was to evaluate the effectiveness and safety of anchoring-EMR (A-EMR).

Methods This was a retrospective analysis of A-EMR procedures for lesions of diameter between 10 and $30 \mathrm{~mm}$ (endoscopic evaluation) performed consecutively in four French centers between May 2017 and January 2018. A-EMR was routinely performed for all EMR using Olympus conventional snares ( 10 or $25 \mathrm{~mm}$ ). The primary outcome was evaluation of the proportion of $\mathrm{R} 0$ resections.

Results A total of 141 A-EMR procedures were performed by 10 operators. Mean lesion size was $19.8 \mathrm{~mm}$. Anchoring was feasible in $96.5 \%$ of cases. There were $81.6 \%$ en bloc resections and $70.2 \%$ R0 resections, with the percentage of procedures decreasing with increasing lesion size $(82.8 \%$ $<20 \mathrm{~mm}, 55.3 \% 21-30 \mathrm{~mm}$, and $50.0 \%>30 \mathrm{~mm}, P=$ $0.002)$. Complete perforations closed endoscopically occurred in $3 / 141$ cases $(2.1 \%)$; none occurred in lesions< $20 \mathrm{~mm}$ in size $(0 / 87)$.

Conclusion The A-EMR technique appears to be promising with a high proportion of R0 for lesions of $10-20 \mathrm{~mm}$ in size without any perforations. It could also offer an alternative to endoscopic submucosal dissection (ESD), or to hybrid techniques to reach R0 for lesions between 20 and $30 \mathrm{~mm}$ in size. 


\section{Introduction}

The quality of endoscopic resections has become a focal point in endoscopy research where the target is techniques with higher rates of complete resection for small polyps (cold snare for lesions $<10 \mathrm{~mm}$ in diameter) or large lesions (endoscopic submucosal dissection (ESD), for large lesions $>30 \mathrm{~mm}$ in size) $[1,2]$. Endoscopic mucosal resection (EMR) with snare is the technique recommended to resect non-invasive colorectal neoplastic lesions $(10-30 \mathrm{~mm})$ effectively and with low morbidity [1]. Nevertheless, such resections are only curative when the lesion is resected in a single specimen (en bloc) with free margins (R0 resection). In the case of piecemeal resection (more than one piece) or in the case of incomplete en bloc resection (undetermined-Rx, or invaded margins-R1), the risk of local recurrence increases and a second colonoscopy is recommended within 6 months to detect and resect potential local recurrence. Thus, improving the quality of EMR to increase the rate of R0 resection and to reduce the need for follow-up procedures should become a target of research. It has been reported that conventional EMR is associated with $62-65 \%$ en bloc resection for lesions $<20 \mathrm{~mm}$ in diameter but $\mathrm{R} 0$ resection has never been evaluated precisely $[1,3,4]$.

We previously described the anchoring EMR (A-EMR) technique [5] which consists of creating a small hole in the submucosa with the tip of a conventional EMR snare to anchor the snare tip in the surrounding margin to reduce sliding and to enlarge the snare opening. This technique is currently used in four centers in France and in this study, we retrospectively assess the current effectiveness and safety of A-EMR.

\section{Methods}

\section{Design}

This was a retrospective multicenter pilot study in four French endoscopy tertiary care centers with experience in EMR and ESD, and where A-EMR was routinely practiced. In total, 10 operators participated in the study. They had each performed $>200$ EMR procedures before beginning A-EMR.

\section{Inclusion criteria}

The study included all consecutive A-EMR procedures attempted between May 2017 and December 2017 with en bloc intent for sessile or flat lesions between 10 and $30 \mathrm{~mm}$ in size. Evaluation of lesion size was done endoscopically; however, size is known to be imprecisely measured this way and may be influenced by the operator's experience. The classic landmark used to help in size evaluation is the comparison with an open biopsy forceps (measuring about $7 \mathrm{~mm}$ in length). Once the specimen was resected, it was systematically stretched on cork using needles and accurately measured.

\section{Exclusion criteria}

The study excluded lesions with pedunculated shape (Ip of Paris classification) or with invasive shape (ulcerated type III of Paris classification), recurrent or residual lesions after previous resection as well as other causes of severe fibrosis (ulcerative co- litis), and lesions with a high risk of superficial adenocarcinoma requiring ESD.

\section{Anchoring-EMR procedure}

In all cases, 10 or $25 \mathrm{~mm}$ conventional snares from Olympus were used (SD-210U, Olympus, Tokyo, Japan) according to lesion size. Snare size was chosen by the operator during the procedure, and use of a distal cap fitted to the colonoscope tip was left to the operator's discretion. After injection of colored fluid (indigo carmine blue, Carmine, SERB, Paris, France) into the submucosal layer, anchoring of the snare tip was performed by creating a small hole in the mucosa a small distance from the lesion edges using electric cutting current (Endocut Q, Erbe, Tübingen, Germany). This incision aimed to reach the submucosa and securely anchor the snare tip there ( $\triangleright$ Fig. 1, $\triangleright$ Video 1 ).

Then the snare was deployed progressively and adjusted around the lesion trying to respect free margins between lesion edges and snare closure. Anchoring allowed the snare to be enlarged after application of pressure ( $\triangleright$ Fig. 1 and $\triangleright$ Fig. 2 ). Once closed, resection was performed as usual with the Endocut $\mathrm{Q}$. Immediately after resection, the resected area was assessed to detect both muscular damage according to Sydney's classification (partial damage with target sign or complete transmural perforation) [6] and residual neoplastic tissue. In the case of perforation, endoscopic closure was attempted. If a single snare EMR resected the whole lesion without residual tissue, resection was considered to be en bloc endoscopically. In the case of residual tissue detected using white light, virtual chromoendoscopy and magnification if needed, additional snare resection(s) (with or without anchoring) was performed leading to a piecemeal resection automatically considered to be R1. After resection, the specimen was stretched on cork with needles and fixed in buffered formalin for pathological assessment. Lesions were then sliced into $2 \mathrm{~mm}$ sections followed by analysis of resection margins to assess R0 status. Pathologists used their conventional technique to analyze the margins and were not aware of the future retrospective evaluation.

\section{Outcomes}

Primary outcome was the proportion of $\mathrm{R} 0$ resections defined histologically by the presence of lateral and deep free margins around the lesion after A-EMR.

Secondary outcomes assessed after A-EMR were: the characteristics of $\mathrm{R} 0$ resection (size, operator, distal cap use); the proportion of successful A-EMR defined by the ability to anchor the snare in the submucosa without slipping of the snare during closure (tip of the snare fixed in the submucosa throughout snare closure); the proportion of en bloc resection defined endoscopically; the proportion of immediate and delayed adverse events (within the first month) including perforation (complete or partial, i.e. with target sign) and bleeding, as well as the proportion of adverse events leading to further surgery. 


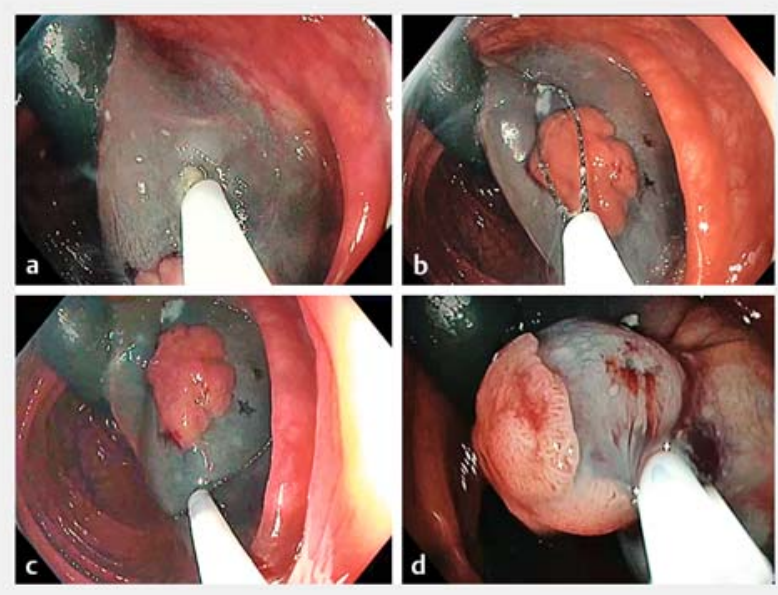

- Fig. 1 Anchoring procedure. First an incision is made into the mucosa using the tip of the snare (a) followed by anchoring of the tip and snare opening (oval shape of snare) (b). Then, pressure on the snare leads to a larger round shape (c) and then resection is performed after snare closure (d).

\section{Data collection}

All data were retrieved from the endoscopy, pathology, and hospitalization reports and were collected, anonymized, and collated in a spreadsheet (Excel; Microsoft, Redmond, WA, United States). Data were verified by an independent research assistant (MMG).

\section{Statistical analysis}

Baseline characteristics and outcome variables were described by the mean, standard deviation (SD) and range for continuous variables, and by frequencies and percentages for categorical variables. Comparisons were performed using Fisher's exact test. Multivariate analysis was performed by penalized logistic regression, including all factors significant in univariate analysis. A $P$ value $<0.05$ was considered to be statistically significant. All analyses were performed using $\mathrm{R}$ software version 3.4.2. (R Core Team. R: A language and environment for statistical computing. R Foundation for Statistical Computing, Vienna, Austria. URL http://www.R-project.org/).

\section{Ethics}

This study was in compliance with the Declaration of Helsinki and received approval from the ethics committee of the Hospices Civils de Lyon (March 7, 2018). All of the patients in the study consented to participate.

\section{Declaration}

This study was declared on the database of the United States National Library of Medicine (clinicaltrials.gov) under the number NCT03467451.



- Fig. 2 Snare shape depending on amount of pressure on the anchoring point. The snare is oval without pressure on the tip (a) and becomes round when a pressure is applied to the tip (b).

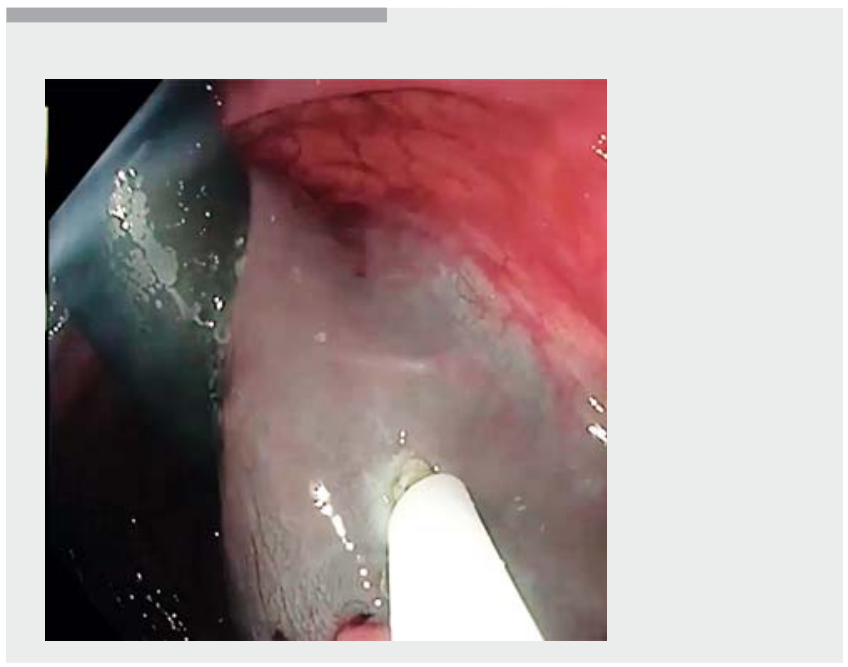

$\checkmark$ Video 1 Snare shape depending on pressure and an example of the procedure. 


\section{Results}

From May 2017 to December 2017, 141 consecutive lesions in 125 patients (mean age: 65.4 years; range $30-89$ years) were resected by 10 operators in four French centers using the A-EMR technique. Among them, 112 patients had 1 lesion (89.6\%), 10 had $2(8.0 \%)$, and 3 patients had 3 lesions $(2.4 \%)$. The size of 6 lesions was not determined pathologically as these were piecemeal resections; the mean (pathologically determined) size of the 135 lesions was $19.8 \mathrm{~mm}$ (range $8-40 \mathrm{~mm}$; SD: 7.1). Lesions were adenomas or intramucosal adenocarcinomas in 89/141 cases (63.1\%), sessile serrated lesions in 51 cases $(36.2 \%)$, and invasive submucosal adenocarcinoma in one case $(0.7 \%)$. All lesions included in the study were endoscopically determined to be between $\geq 10$ and $\leq 30 \mathrm{~mm}$; according to pathological assessment, there were 87 lesions between 8 and $20 \mathrm{~mm}, 38$ lesions between 21 and $30 \mathrm{~mm}$, and 10 lesions $>30 \mathrm{~mm}$; the characteristics of the resected lesions are presented in $>$ Table 1.

\section{Technical results}

The injection before EMR was performed with saline serum in 113 cases $(80.1 \%)$, with hyaluronic acid $(0.4 \%$ solution; Sigmavisc, Life Partners Europe, Paris, France) in 24 (17.0\%), and with a glycerol mixture in 4 cases $(2.8 \%)$. Snare anchoring through the mucosa was feasible in 136 cases (96.5\%). In five cases, anchoring was not feasible since the snare slipped away from the anchoring point before complete snare closure. A cap was fitted to the tip of the colonoscope in 54 cases (38.3\%), no cap was used in 54 cases (38.3\%), and data were missing in 33 cases (23.4\%). A $25 \mathrm{~mm}$ snare was used in 127 cases (90.1\%) and a $10 \mathrm{~mm}$ snare in 14 cases (9.9\%). Endoscopically, en bloc resection was performed in $115 / 141$ cases (81.6\%).

\section{Histology results}

R0 resections were obtained in 99/141 cases (70.2\%). The proportion of R0 resections obtained was significantly different according to lesion large diameter: $82.8 \%$ (72/87) for lesions $<20 \mathrm{~mm}$ in size, $55.3 \%(21 / 38)$ for lesions between 20 and $30 \mathrm{~mm}$ in size, and $50.0 \%(5 / 10)$ for those $>30 \mathrm{~mm}$ in size $(P=$ 0.002). When anchoring was attempted but not achieved ( 5 cases), the proportion of $\mathrm{R} 0$ resections was $0 \%$ although the proportion obtained when anchoring was feasible was $72.8 \%$ and the difference was statistically significant $(P=0.002)$. There was no statistically significant difference in the proportion of R0 resections according to the other factors investigated, including operator or type of solution injected into the submucosa ( $\triangleright$ Table 2). There were three operators with a proportion of R0 below the overall value (70.2\%) and two who attained $100 \%$ R0. In multivariate analysis adjusted for the size of the lesion, the effect of the success of anchoring was still significant ( $P$ value $=0.025$ ).

\section{Adverse events}

Perforations occurred in 5/141 resections (3.5\%) in 125 patients $(5 / 125,4.0 \%)$ including complete transmural perforation in three cases $(2.1 \%)$ and partial perforation with target sign in
- Table 1 Characteristics of lesions resected by A-EMR.

\begin{tabular}{|c|c|}
\hline Characteristics & Lesions, $n=141$ \\
\hline \multicolumn{2}{|l|}{ Location } \\
\hline - Caecum & $24(17.0)$ \\
\hline - Right colon & $53(37.6)$ \\
\hline - Right angle & $9(6.4)$ \\
\hline - Transverse colon & $16(11.3)$ \\
\hline - Left angle & $13(9.2)$ \\
\hline - Sigmoid colon & $15(10.6)$ \\
\hline - Rectum & $11(7.8)$ \\
\hline - Missing data & 0 \\
\hline \multicolumn{2}{|l|}{ Pathologically-determined size } \\
\hline - $8-20 \mathrm{~mm}$ & $87(64.4)$ \\
\hline - $21-30 \mathrm{~mm}$ & $38(28.1)$ \\
\hline - $>30 \mathrm{~mm}$ & $10(7.4)$ \\
\hline - Missing data & 6 \\
\hline \multicolumn{2}{|l|}{ Paris classification } \\
\hline . 0 -Is & $52(36.9)$ \\
\hline - 0 -Isp & $2(1.4)$ \\
\hline - 0-lia & $67(47.5)$ \\
\hline - 0 -lib & $5(3.5)$ \\
\hline . 0 -lic & $1(0.7)$ \\
\hline . 0 -lla+lic & $3(2.1)$ \\
\hline . $0-11 a+$ Is & $11(7.8)$ \\
\hline - Missing data & 0 \\
\hline \multicolumn{2}{|l|}{ Histology subtype } \\
\hline - Adenoma or mucosal adenocarcinoma & $89(63.1)$ \\
\hline - Sessile serrated lesion & $51(36.2)$ \\
\hline - Submucosal invasive adenocarcinoma & $1(0.7)$ \\
\hline - Missing data & 0 \\
\hline
\end{tabular}

two cases (1.4\%). Complete perforations never occurred for lesions $<20 \mathrm{~mm}$ in size $(0 / 87,0 \%)$, in one case for lesions between 20 and $30 \mathrm{~mm}$ in size $(1 / 38,2.6 \%)$, and in two cases $>30 \mathrm{~mm}$ in size $(2 / 10,20 \%)$. All were treated conservatively with endoscopic closure using hemoclips, and none led to salvage surgery. One delayed bleeding (melena) occurred after 24 hours $(0.7 \%)$ and which stopped spontaneously without a new colonoscopy; blood transfusion was not necessary.

\section{Discussion}

Anchoring-EMR is effective in achieving en bloc and R0 resections of colorectal neoplasia lesions, in particular, those $<20 \mathrm{~mm}$ in size. The results presented herein for the A-EMR 
- Table 2 En bloc and R0 resection according to lesion and procedure characteristics.

\begin{tabular}{|c|c|c|c|c|}
\hline Characteristics & $\mathbf{n}$ & En bloc resection (\%) & R0 resection (\%) & $P$ value \\
\hline \multicolumn{4}{|l|}{ Success of anchoring } & 0.002 \\
\hline - Yes & 136 & $115(84.6)$ & $99(72.8)$ & \\
\hline - No & 5 & $0(0.0)$ & $0(0.0)$ & \\
\hline - Missing data & 0 & 0 & 0 & \\
\hline \multicolumn{4}{|l|}{ Pathology-determined size } & 0.002 \\
\hline - $8-20 \mathrm{~mm}$ & 87 & $80(92.0)$ & $72(82.8)$ & \\
\hline - $21-30 \mathrm{~mm}$ & 38 & $26(68.4)$ & $21(55.3)$ & \\
\hline . $>30 \mathrm{~mm}$ & 10 & $8(80.0)$ & $5(50.0)$ & \\
\hline - Missing data & 6 & 6 & 6 & \\
\hline \multicolumn{4}{|l|}{ Center } & 0.737 \\
\hline . 1 & 41 & $33(80.5)$ & $27(65.9)$ & \\
\hline .2 & 55 & $47(85.5)$ & $38(69.1)$ & \\
\hline .3 & 21 & $16(76.2)$ & $15(71.4)$ & \\
\hline .4 & 24 & $19(79.2)$ & $19(79.2)$ & \\
\hline - Missing data & 0 & 0 & 0 & \\
\hline \multicolumn{4}{|l|}{ Operator } & 0.399 \\
\hline . 1 & 5 & $5(100)$ & $5(100)$ & \\
\hline .2 & 7 & $5(71.4)$ & $5(71.4)$ & \\
\hline .3 & 13 & $9(69.2)$ & $6(46.2)$ & \\
\hline .4 & 12 & $10(83.3)$ & $6(50.0)$ & \\
\hline .5 & 12 & $10(83.3)$ & $9(75.0)$ & \\
\hline .6 & 21 & $16(76.2)$ & $15(71.4)$ & \\
\hline .7 & 21 & $19(90.5)$ & $16(76.2)$ & \\
\hline .8 & 25 & $21(84.0)$ & $17(68.0)$ & \\
\hline .9 & 24 & $19(79.2)$ & $19(79.2)$ & \\
\hline - 10 & 1 & $1(100)$ & $1(100.0)$ & \\
\hline - Missing data & 0 & 0 & 0 & \\
\hline \multicolumn{4}{|l|}{ Histology subtype } & 0.339 \\
\hline - Adenoma/carcinoma & 90 & $70(77.8)$ & $66(73.3)$ & \\
\hline - Sessile serrated lesion & 51 & $45(88.2)$ & $33(64.7)$ & \\
\hline - Missing data & 0 & 0 & 0 & \\
\hline \multicolumn{4}{|l|}{ Cap assisted } & 0.283 \\
\hline - Yes & 54 & $45(83.3)$ & $42(77.8)$ & \\
\hline - No & 54 & $44(81.5)$ & $36(66.7)$ & \\
\hline - Missing data & 33 & 33 & 33 & \\
\hline \multicolumn{4}{|l|}{ Injection medium } & 0.671 \\
\hline - Saline & 113 & $93(82.3)$ & $77(68.1)$ & \\
\hline - Glycerol mixture & 4 & $3(75.0)$ & $3(75.0)$ & \\
\hline - Hyaluronic acid preparation & 24 & $19(79.2)$ & $19(79.2)$ & \\
\hline - Missing data & 0 & 0 & 0 & \\
\hline
\end{tabular}


technique compare well with the literature as it is reported that only $62-65 \%$ of resections are en bloc with conventional EMR $[1,3,4,7]$; interestingly, it is difficult to compare $R 0$ resection rates as they do not seem to be reported in studies evaluating EMR.

Current quality indicators for endoscopic resections require en bloc resection for small polyps $(<10 \mathrm{~mm})$, and on this basis, cold snaring of these is strongly recommended [1]. For larger lesions (>30 mm), EMR is mostly piecemeal in intent, whereas ESD has a high $\mathrm{R} 0$ resection rate [8-11]. Nevertheless, ESD is time consuming and requires expensive devices and thorough training. Alternative hybrid strategies have been developed to push the boundaries of ESD indications, but the resulting $\mathrm{R} 0$ resection rate was far from perfect and, although marginally quicker, these use the same devices [12] which does not meaningfully change the cost.

The A-EMR technique associates a simple technical trick with conventional EMR snares and with a good overall proportion of en bloc and $\mathrm{R} 0$ resections in different colon segments, including more than $60 \%$ of resections in the ascending colon, known to be a technically challenging location. In this study, when considering only lesions $<20 \mathrm{~mm}$ in size, which are the most suitable for en bloc EMR according to ESGE guidelines [1], AEMR achieved a high quality level as the proportion of R0 was comparable to that reported for ESD in Japanese studies [8, $13,14]$, but without any perforations. This is of interest because, as in the case of $\mathrm{R} 0$ resections for non-invasive neoplasia, the risk of local recurrence is theoretically null and therefore there is probably no need for follow-up colonoscopy to detect local recurrence [15] despite the recent ESGE guidelines [16].

For larger lesions, although there was no comparator group, the proportion of R0 may be better than for conventional EMR, but does not reach that obtained with ESD, and it is of note that perforations occurred in such cases. Among these, the proportion of perforations was $6.2 \%$, which is considerably higher than the $1.2 \%$ reported after conventional EMR in comparably sized lesions [17]. Anchoring could lead to a deeper catching and, as a result, to an increased risk of perforation, but comparative data are needed to evaluate the morbidity of this technique compared to conventional EMR. The latter point highlights the main limitation of this study.

A general point to consider is that, in light of the relatively high perforation rate in this series, operators using A-EMR should be careful if en bloc resection is attempted for a lesion $>2 \mathrm{~cm}$ in size. Furthermore, the number of procedures for each operator was low, which precluded analysis of a potential operator effect on the R0 result. This was further compounded by the non-standardized solution used for the submucosal cushion and the non-systematic use of a distal cap. Although not significant in this sample, a larger difference between the frequency of en bloc (88.2\%) and R0 resections (64.7\%) appeared for sessile serrated lesions than for adenomas (respectively, 77.8 and $73.3 \%$ ). This larger difference underlines the difficult delineation of sessile serrated lesions endoscopically and then the technical challenge to remove those lesions with free margins as previously reported [18]; this could affect the results in a future comparative study. Another point to consider is that the expertise of the physicians involved is also a limitation since most of them were also expert in ESD and were used to accessing the submucosa, which may affect the generalizability of the results.

Our opinion is that the main limitation of conventional EMR is the lack of snare fixation at the distal point with a risk of snare sliding to the lesion edge leading to incomplete (R1), uncertain $(\mathrm{Rx})$ or piecemeal resections. This is a postulation that led us to create and develop this method of A-EMR.

To summarize, the A-EMR technique appears to be promising with a high proportion of R0 for lesions of 10 to $20 \mathrm{~mm}$ diameter without any perforation. It could also offer an alternative to ESD or to hybrid techniques to reach R0 for lesions between 20 and $30 \mathrm{~mm}$ saving time and money by using conventional devices. Randomized comparative studies are required using standardized procedures to conclude as to the potential benefits of this technique.

\section{Acknowledgments}

We sincerely thank Pr Jean-François Bretagne for reviewing this article.

\section{Competing interests}

None

\section{References}

[1] Ferlitsch M, Moss A, Hassan C et al. Colorectal polypectomy and endoscopic mucosal resection (EMR): European Society of Gastrointestinal Endoscopy (ESGE) Clinical Guideline. Endoscopy 2017; 49: $270-297$

[2] Tanaka S, Kashida H, Saito Y et al. JGES guidelines for colorectal endoscopic submucosal dissection/endoscopic mucosal resection. Dig Endosc 2015; 27: $417-434$

[3] Yoshida N, Naito Y, Inada Y et al. Multicenter study of endoscopic mucosal resection using $0.13 \%$ hyaluronic acid solution of colorectal polyps less than $20 \mathrm{~mm}$ in size. Int J Colorectal Dis 2013; 28: 985 - 991

[4] Woodward T, Crook JE, Raimondo M et al. Improving complete EMR of colorectal neoplasia: a randomized trial comparing snares and injectate in the resection of large sessile colon polyps. Gastrointest Endosc 2015; 81: 673-681

[5] Jacques ], Legros R, Charissoux A et al. Anchoring the snare tip by means of a small incision facilitates en bloc endoscopic mucosal resection and increases the specimen size. Endoscopy 2017; 49: E39E41

[6] Burgess NG, Bassan MS, McLeod D et al. Deep mural injury and perforation after colonic endoscopic mucosal resection: a new classification and analysis of risk factors. Gut 2017; 66: 1779-1789

[7] Yoshida N, Naito Y, Inada Y et al. Endoscopic mucosal resection with $0.13 \%$ hyaluronic acid solution for colorectal polyps less than $20 \mathrm{~mm}$ : a randomized controlled trial. J Gastroenterol Hepatol 2012; 27: $1377-1383$

[8] Saito Y, Fukuzawa M, Matsuda T et al. Clinical outcome of endoscopic submucosal dissection versus endoscopic mucosal resection of large colorectal tumors as determined by curative resection. Surg Endosc 2010; $24: 343-352$ 
[9] Fujiya M, Tanaka K, Dokoshi T et al. Efficacy and adverse events of EMR and endoscopic submucosal dissection for the treatment of coIon neoplasms: a meta-analysis of studies comparing EMR and endoscopic submucosal dissection. Gastrointest Endosc 2015; 81: 583 595

[10] Wang J, Zhang X-H, Ge J et al. Endoscopic submucosal dissection vs endoscopic mucosal resection for colorectal tumors: A meta-analysis. World J Gastroenterol 2014; 20: $8282-8287$

[11] Kobayashi N, Yoshitake N, Hirahara Y et al. Matched case-control study comparing endoscopic submucosal dissection and endoscopic mucosal resection for colorectal tumors. J Gastroenterol Hepatol 2012; $27: 728-733$

[12] Toyonaga T, Man-i M, Chinzei R et al. Endoscopic treatment for early stage colorectal tumors: the comparison between EMR with small incision, simplified ESD, and ESD using the standard flush knife and the ball tipped flush knife. Acta Chir lugosl 2010; 57: 41-46

[13] Saito Y, Uraoka T, Yamaguchi Y et al. A prospective, multicenter study of 1111 colorectal endoscopic submucosal dissections (with video). Gastrointest Endosc 2010; 72: 1217-1225
[14] Watanabe T, Itabashi M, Shimada Y et al. Japanese Society for Cancer of the Colon and Rectum. Japanese Society for Cancer of the Colon and Rectum (JSCCR) Guidelines 2014 for treatment of colorectal cancer. Int J Clin Oncol 2015; 20: 207-239

[15] Pioche M, Walter T. Endoscopic removal of colorectal T1 cancers: Why is a 1-year follow-up recommended by ESGE when resection is R0 and curative? Endosc Int Open 2019; 7: E816 - E817

[16] Hassan C, Wysocki PT, Fuccio L et al. Endoscopic surveillance after surgical or endoscopic resection for colorectal cancer: European Society of Gastrointestinal Endoscopy (ESGE) and European Society of Digestive Oncology (ESDO) Guideline. Endoscopy 2019; 51: 266 - 277

[17] Bronsgeest K, Huisman JF, Langers A et al. Safety of endoscopic mucosal resection (EMR) of large non-pedunculated colorectal adenomas in the elderly. Int J Colorectal Dis 2017; 32: 1711-1717

[18] Pohl H, Srivastava A, Bensen SP et al. Incomplete polyp resection during colonoscopy - results of the complete adenoma resection (CARE) study. Gastroenterology 2013; 144: $74-80 . e 1$ 